\title{
Zeittafel zum Leben und Schaffen von Carl Friedrich Gauß
}

30.4.1777

1784-1788

1788-1792

1792-1795

1795-1798

1796

1798-1807

16.7.1799

1801

Oktober 1801

31.1./12.2.1802

13.11.1802

9.10.1805

21.8.1806

25.7.1807

1807

11.10.1809

1809

4.8.1810 geboren in Braunschweig

Besuch der Katharinen-Volksschule in Braunschweig

Besuch des Gymnasiums Catharineum in Braunschweig

Studium am Collegium Carolinum in Braunschweig

Studium an der Georgia Augusta in Göttingen mit einem

Stipendium des Herzogs von Braunschweig

Entdeckung der Konstruierbarkeit des regelmäßigen Siebzehnecks

Privatgelehrter in Braunschweig mit einem herzoglichen Stipendium

Promotion in Helmstedt in absentia bei Johann Friedrich Pfaff mit dem ersten vollständigen Beweis des Fundamentalsatzes der Algebra

Veröffentlichung der „Disquisitiones arithmeticae“

Berechnung der Bahn des von Giuseppe Piazzi am 1.1.1801 entdeckten kleinen Planeten Ceres

Korrespondierendes Mitglied der Kaiserlichen Akademie der Wissenschaften zu St. Petersburg

Korrespondierendes Mitglied der Königlichen Societät der Wissenschaften zu Göttingen

Heirat mit Johanna Osthoff aus Braunschweig

Geburt des ersten Sohnes Joseph

Ernennung zum Ordentlichen Professor für Astronomie und Direktor der Universitätssternwarte in Göttingen

Ordentliches Mitglied der Königlichen Societät der Wissenschaften zu Göttingen

Tod der ersten Frau Johanna

Veröffentlichung des astronomischen Hauptwerks „Theoria motus"

Heirat mit Friederica Wilhelmine Waldeck 
18.4.-23.5.1816 Reise mit Sohn Joseph nach Gotha und München

Oktober $1816 \quad$ Umzug in die neue Sternwarte in Göttingen

9.5.1820

1824

1828

Auftrag, die Triangulation des Königreichs Hannover vorzunehmen

Erfindung des Heliotropen

Ehrenmitglied der Kaiserlichen Akademie der Wissenschaften zu St. Petersburg

Veröffentlichung der „Disquisitiones generales circa superficies curvas"
14.9.-3.10.1828 Persönlicher Gast bei Alexander von Humboldt in Berlin, Teilnahme an der 7. Versammlung deutscher Naturforscher und Ärzte

April 1831

12.9.1831

15.12 .1832

$1834 / 1836$

19.9.1837

18.11.1837

$1843-1849$

$1844 / 1847$

16.7.1849

23.2.1855

Wilhelm Weber erhält einen Ruf an die Universität Göttingen

Tod der zweiten Frau

Gauß stellt seine „Intensitas“ in der Göttinger Societät der Wissenschaften vor

Erfindung des elektromagnetischen Telegraphen gemeinsam mit Weber

Bau des Magnetischen Observatoriums in Göttingen

Gründung des Magnetischen Vereins zu Göttingen

Feier des 100-jährigen Bestehens der Universität Göttingen

Protest der „Göttinger Sieben“ gegen die Aufhebung der Verfassung

Veröffentlichung der „Allgemeinen Theorie des Erdmagnetismus“"

Wilhelm Weber in Leipzig tätig, kehrt dann nach Göttingen zurück

Veröffentlichung der ersten und der zweiten Abhandlung der „Untersuchungen über Gegenstände der höhern Geodäsie“"

Feier des goldenen Doktorjubiläums in Göttingen

gestorben in Göttingen 